\title{
Mechanotransduction in Ischemic Cardiac Tissue: A Mechanical Bidomain Approach under Plane Stress
}

\author{
Austin Fee* \& Bradley J. Roth \\ *Department of Physics, Oakland University, Rochester, Michigan \\ https://doi.org/10.33697/ ajur.2019.001
}

Student: austinfee@oakland.edu*

Mentor:roth@oakland.edu

\begin{abstract}
Mechanotransduction is the process by which biological tissue translates mechanical forces and signals, such as those produced by strains or membrane forces, into biological reactions including cell remodeling, growth, and differentiation. While some analyses assume strain (the derivative of either the intracellular or extracellular displacement) as the cause of mechanotransduction, this paper assumes that differences between the intracellular and extracellular displacements, known as membrane force, result in mechanical forces acting on integrin proteins, causing mechanotransduction. The mechanical bidomain model is a twodimensional mathematical representation that describes this behavior. Previous analyses describe mechanotransduction using plane strain, which assumes zero displacement in the z-direction. This analysis uses plane stress, which assumes zero stress in the z-direction, to describe where mechanotransduction occurs in comparison to plane strain models. A sample of healthy tissue with a circular ischemic region with no active tension in the center is analyzed using numerical methods. Fixed and free boundary conditions are implemented. Under fixed conditions, the membrane force was largest in the ischemic border zone and zero everywhere else. However, the strain was found to be largest in the ischemic region. Under free conditions, the membrane force was largest on the vertical edges and in the ischemic border zone. The strain was found to be nearly zero in the ischemic region and ranged up to $10 \%$ throughout the tissue. In conclusion, this paper found that both plane strain and plane stress predict a membrane force in the ischemic border zone, but the distribution of individual displacements and strain vary according to each model. These results are significant in determining which model is most appropriate to use in predicting how mechanical forces affect cellular remodeling when analyzing thin monolayers of tissue.
\end{abstract}

\section{KEYWORDS}

Mechanotransduction; Plane Stress; Tissue Engineering; Mechanical Bidomain Model; Biomechanics; Strain; Intracellular Cytoskeleton; Extracellular Matrix; Ischemia; Finite Differences

\section{INTRODUCTION}

When cardiac tissue is subjected to a force, the individual cells react and change via a process called mechanotransduction, which is a cell's biological response to a mechanical stimulus. More specifically, integrin proteins, connecting the intracellular cytoskeleton and the extracellular matrix, are predicted to be the components that induce mechanotransduction when a force causes a difference in displacement between the intra- and extracellular spaces. ${ }^{1}$ In effect, the growth, remodeling, and differentiation of the cells have been hypothesized as being the results of mechanotransduction. ${ }^{2}$ To predict and describe this behavior, a mathematical model called the mechanical bidomain model has been developed. ${ }^{3}$ This model is macroscopic in that it relates the interactions between the intracellular and extracellular spaces separately through forces on integrin membrane proteins (the membrane force). ${ }^{3,4}$ The model also predicts the stress (force per unit area) and strain (change in length divided by the original length) existing throughout both the cytoskeleton and the extracellular matrix.

In previous models, the two-dimensional case of plane strain was analyzed; it assumed no tissue displacements perpendicular ( $z$ direction) to the tissue. ${ }^{3}$ However, numerous experiments are conducted on thin monolayers of cells that can experience displacement in all directions. For the case of plane stress, in comparison, displacements can occur in the z-direction, but no stress exists in this direction because there is no force acting normal to the cell monolayers. ${ }^{5}$ This analysis examines a rectangular sheet of tissue with a circular ischemic (oxygen deprived) region in the center, similar to that examined by Gandhi and Roth for plane strain. ${ }^{6}$ The purpose of this paper is to analyze mechanotransduction under plane stress and compare the results to the previous analysis under plane strain to determine possible implications of the effects of mechanotransduction in cardiac tissue. Our mathematical simulations of mechanotransduction around a region of ischemia may provide insight into how cardiac tissue remodels following a heart attack. 


\section{METHODS AND PROCEDURES}

The mechanical bidomain model was used to describe the behavior of the mechanical force, in the form of stresses and strains, acting upon a sheet of cardiac tissue. This model was used to derive equations with plane stress, which assumes that the tissue has zero stress in the $z$-direction and is incompressible (no change in volume). Stress $(\tau)$ and strain $(\varepsilon)$ are related by

$$
\begin{array}{ccc}
\tau_{i x x}=-p+2 v \varepsilon_{i x x}+T & \tau_{i y y}=-p+2 v \varepsilon_{i y y} & \tau_{i z z}=-p+2 v \varepsilon_{i z z} \\
\tau_{i x y}=2 v \varepsilon_{i x y} & \tau_{i x z}=2 v \varepsilon_{i x z} & \tau_{i y z}=2 v \varepsilon_{i y z},
\end{array}
$$

Equation 1.

where $p$ is the intracellular hydrostatic pressure, $v$ is the intracellular shear modulus relating the strain to the stress, and $T$ is the active tension produced by actin and myosin molecules that act in the direction along the fibers ( $x$-direction). The intracellular strains are defined with respect to the intracellular tissue displacement, $\mathbf{u}$, by

$$
\begin{array}{ccc}
\varepsilon_{i x x}=\frac{\partial u_{x}}{\partial x} & \varepsilon_{i y y}=\frac{\partial u_{y}}{\partial y} & \varepsilon_{i z z}=\frac{\partial u_{z}}{\partial z} \\
\varepsilon_{i x y}=\frac{1}{2}\left(\frac{\partial u_{x}}{\partial y}+\frac{\partial u_{y}}{\partial x}\right) & \varepsilon_{i x z}=\frac{1}{2}\left(\frac{\partial u_{x}}{\partial z}+\frac{\partial u_{z}}{\partial x}\right) & \varepsilon_{i y z}=\frac{1}{2}\left(\frac{\partial u_{y}}{\partial z}+\frac{\partial u_{z}}{\partial y}\right) .
\end{array}
$$

Equation 2.

Similar equations exist for the extracellular space,

$$
\begin{array}{ccc}
\tau_{\text {exx }}=-q+2 \mu \varepsilon_{\text {exx }} & \tau_{\text {eyy }}=-q+2 \mu \varepsilon_{\text {eyy }} & \tau_{\text {ezz }}=-q+2 \mu \varepsilon_{\text {ezz }} \\
\tau_{\text {exy }}=2 \mu \varepsilon_{\text {exy }} & \tau_{\text {exz }}=2 \mu \varepsilon_{\text {exz }} & \tau_{\text {eyz }}=2 \mu \varepsilon_{\text {eyz }},
\end{array}
$$

Equation 3.

and

$$
\begin{array}{ccc}
\varepsilon_{\text {exx }}=\frac{\partial w_{x}}{\partial x} & \varepsilon_{\text {eyy }}=\frac{\partial w_{y}}{\partial y} & \varepsilon_{\text {ezz }}=\frac{\partial w_{z}}{\partial z} \\
\varepsilon_{\text {exy }}=\frac{1}{2}\left(\frac{\partial w_{x}}{\partial y}+\frac{\partial w_{y}}{\partial x}\right) & \varepsilon_{\text {exz }}=\frac{1}{2}\left(\frac{\partial w_{x}}{\partial z}+\frac{\partial w_{z}}{\partial x}\right) & \varepsilon_{\text {eyz }}=\frac{1}{2}\left(\frac{\partial w_{y}}{\partial z}+\frac{\partial w_{z}}{\partial y}\right),
\end{array}
$$

Equation 4.

where $\mathbf{w}$ is the extracellular displacement, $q$ is the extracellular hydrostatic pressure, and $\mu$ is the extracellular shear modulus accounting for the elastic properties of the extracellular matrix. Incompressibility implies that

$$
\begin{gathered}
\varepsilon_{i x x}+\varepsilon_{i y y}+\varepsilon_{i z z}=0 \\
\varepsilon_{\text {exx }}+\varepsilon_{\text {eyy }}+\varepsilon_{\text {ezz }}=0 .
\end{gathered}
$$

Equation 5.

Equation 6. 
The incompressibility condition indicates that a shortening in one direction must be accompanied by a lengthening in another direction. Since plane stress means the stresses normal to the tissue are zero, $\tau_{i z z}=\tau_{i x z}=\tau_{i y z}=\tau_{\text {ezz }}=\tau_{\text {exz }}=\tau_{\text {eyz }}=0$. One implication of plane stress is that $p=-2 v\left(\varepsilon_{i x x}+\varepsilon_{\text {iyy }}\right)$ and $q=-2 \mu\left(\varepsilon_{\text {exx }}+\varepsilon_{\text {eyy }}\right)$. Plugging $q$ and $p$ into Equation 1 and Equation 3, the intra- and extracellular stresses become

$$
\begin{aligned}
& \tau_{i x x}=2 v\left[2 \frac{\partial u_{x}}{\partial x}+\frac{\partial u_{y}}{\partial y}\right]+T \quad \tau_{i y y}=2 v\left[2 \frac{\partial u_{y}}{\partial y}+\frac{\partial u_{x}}{\partial x}\right] \quad \tau_{i x y}=v\left[\frac{\partial u_{x}}{\partial y}+\frac{\partial u_{y}}{\partial x}\right] \\
& \tau_{\text {exx }}=2 \mu\left[2 \frac{\partial w_{x}}{\partial x}+\frac{\partial w_{y}}{\partial y}\right] \quad \tau_{\text {eyy }}=2 \mu\left[2 \frac{\partial w_{y}}{\partial y}+\frac{\partial w_{x}}{\partial x}\right] \quad \tau_{\text {exy }}=\mu\left[\frac{\partial w_{x}}{\partial y}+\frac{\partial w_{y}}{\partial x}\right] . \quad \text { Equation 8. }
\end{aligned}
$$

The equations of static equilibrium are

$$
\begin{gathered}
\frac{\partial \tau_{i x x}}{\partial x}+\frac{\partial \tau_{i x y}}{\partial y}=K\left(u_{x}-w_{x}\right) \\
\frac{\partial \tau_{i y y}}{\partial y}+\frac{\partial \tau_{i x y}}{\partial x}=K\left(u_{y}-w_{y}\right) \\
\frac{\partial \tau_{\text {exx }}}{\partial x}+\frac{\partial \tau_{\text {exy }}}{\partial y}=-K\left(u_{x}-w_{x}\right) \\
\frac{\partial \tau_{\text {eyy }}}{\partial y}+\frac{\partial \tau_{\text {exy }}}{\partial x}=-K\left(u_{y}-w_{y}\right),
\end{gathered}
$$

Equation 9.

Equation 10.

Equation 11.

Equation 12.

where $K$ is a Hookean spring constant used to account for the elasticity of the integrins coupling the intra- and extracellular spaces. ${ }^{3}$ The active tension $(T)$ is assumed to be uniform throughout the tissue excluding the circular ischemic region defined by $R=\sqrt{x^{2}+y^{2}}<a$ where $T=0$. We chose this geometry and fiber orientation because it is identical to the one analyzed by Gandhi and Roth. ${ }^{6}$

The boundary conditions at the outer edge of the tissue were either fixed or free. Initially, the boundaries were fixed such that the displacements $\mathbf{u}=\mathbf{w}=0$, as was analyzed by Gandhi and Roth. ${ }^{6}$ Alternatively, the boundaries were also modeled as being free such that the stress normal to the boundary is zero, as was analyzed by Sharma. ${ }^{7}$

Differentiating Equation 7 and Equation 8 and substituting them into Equations 9-12 yields

$$
\begin{gathered}
v\left[4 \frac{\partial^{2} u_{x}}{\partial x^{2}}+3 \frac{\partial^{2} u_{y}}{\partial x \partial y}+\frac{\partial^{2} u_{x}}{\partial y^{2}}\right]+\frac{d T}{d x}=K\left(u_{x}-w_{x}\right) \\
v\left[4 \frac{\partial^{2} u_{y}}{\partial y^{2}}+3 \frac{\partial^{2} u_{x}}{\partial x \partial y}+\frac{\partial^{2} u_{y}}{\partial x^{2}}\right]=K\left(u_{y}-w_{y}\right) \\
\mu\left[4 \frac{\partial^{2} w_{x}}{\partial x^{2}}+3 \frac{\partial^{2} w_{y}}{\partial x \partial y}+\frac{\partial^{2} w_{x}}{\partial y^{2}}\right]=-K\left(u_{x}-w_{x}\right) \\
\mu\left[4 \frac{\partial^{2} w_{y}}{\partial y^{2}}+3 \frac{\partial^{2} w_{x}}{\partial x \partial y}+\frac{\partial^{2} w_{y}}{\partial x^{2}}\right]=-K\left(u_{y}-w_{y}\right) .
\end{gathered}
$$

Equation 13.

Equation 14.

Equation 15.

Equation 16. 
To solve Equations 13-16 numerically, the derivatives are replaced by finite differences and solved iteratively using overrelaxation. ${ }^{6}$ The tissue sheet is described as a grid of points with $i=1,2, \ldots, N_{x}$ in the $x$-direction and $j=1,2, \ldots, N_{y}$ in the $y$ direction. Solving Equations 13-16 for $\left.u_{x}\left(i_{j}\right), u_{y}\left(i_{j}\right)\right), w_{x}\left(i_{j}\right)$, and $w_{y}\left(i_{j}\right)$ in terms of their nearest neighbors, we obtained:

$$
\begin{aligned}
& u_{x}(i, j)=\frac{\lambda \omega_{x}\left(\frac{\Delta^{2} \lambda}{\sigma^{2}(1+\lambda)}\right)+\left(\frac{\Delta^{2} \lambda}{\sigma^{2}(1+\lambda)}+10 \lambda\right)\left(\theta_{x}+\frac{\Delta T_{x}}{2 \nu}\right)}{\left(\frac{\Delta^{2} \lambda}{\sigma^{2}(1+\lambda)}+10 \lambda\right)\left(\frac{\Delta^{2} \lambda}{\sigma^{2}(1+\lambda)}+10\right)-\left(\frac{\Delta^{4} \lambda^{2}}{\sigma^{4}(1+\lambda)^{2}}\right)} \\
& w_{x}(i, j)=\frac{\lambda \omega_{x}\left(\frac{\Delta^{2} \lambda}{\sigma^{2}(1+\lambda)}+10\right)+\left(\frac{\Delta^{2} \lambda}{\sigma^{2}(1+\lambda)}\right)\left(\theta_{x}+\frac{\Delta T_{x}}{2 v}\right)}{\left(\frac{\Delta^{2} \lambda}{\sigma^{2}(1+\lambda)}+10 \lambda\right)\left(\frac{\Delta^{2} \lambda}{\sigma^{2}(1+\lambda)}+10\right)-\left(\frac{\Delta^{4} \lambda^{2}}{\sigma^{4}(1+\lambda)^{2}}\right)} \\
& u_{y}(i, j)=\frac{\lambda \omega_{y}\left(\frac{\Delta^{2} \lambda}{\sigma^{2}(1+\lambda)}\right)+\left(\frac{\Delta^{2} \lambda}{\sigma^{2}(1+\lambda)}+10 \lambda\right) \theta_{y}}{\left(\frac{\Delta^{2} \lambda}{\sigma^{2}(1+\lambda)}+10 \lambda\right)\left(\frac{\Delta^{2} \lambda}{\sigma^{2}(1+\lambda)}+10\right)-\left(\frac{\Delta^{4} \lambda^{2}}{\sigma^{4}(1+\lambda)^{2}}\right)} \\
& w_{y}(i, j)=\frac{\lambda \omega_{y}\left(\frac{\Delta^{2} \lambda}{\sigma^{2}(1+\lambda)}+10\right)+\left(\frac{\Delta^{2} \lambda}{\sigma^{2}(1+\lambda)}\right) \theta_{y}}{\left(\frac{\Delta^{2} \lambda}{\sigma^{2}(1+\lambda)}+10 \lambda\right)\left(\frac{\Delta^{2} \lambda}{\sigma^{2}(1+\lambda)}+10\right)-\left(\frac{\Delta^{4} \lambda^{2}}{\sigma^{4}(1+\lambda)^{2}}\right)}
\end{aligned}
$$

Equation 17.

Equation 18.

Equation 19.

Equation 20.

where,

$$
\begin{aligned}
& \theta_{x}=4\left[u_{x}(i+1, j)+u_{x}(i-1, j)\right]+\frac{3}{4}\left[u_{y}(i+1, j+1)+u_{y}(i-1, j-1)-u_{y}(i-1, j+1)-u_{y}(i+1, j-1)\right]+ \\
& u_{x}(i, j+1)+u_{x}(i, j-1) \\
& \omega_{x}=4\left[w_{x}(i+1, j)+w_{x}(i-1, j)\right]+\frac{3}{4}\left[w_{y}(i+1, j+1)+w_{y}(i-1, j-1)-w_{y}(i-1, j+1)-w_{y}(i+1, j-1)\right]+ \\
& w_{x}(i, j+1)+w_{x}(i, j-1) \\
& \theta_{y}=4\left[u_{y}(i, j+1)+u_{y}(i, j-1)\right]+\frac{3}{4}\left[u_{x}(i+1, j+1)+u_{x}(i-1, j-1)-u_{x}(i-1, j+1)-u_{x}(i+1, j-1)\right]+ \\
& u_{y}(i+1, j)+u_{y}(i-1, j) \\
& \omega_{y}=4\left[w_{y}(i, j+1)+w_{y}(i, j-1)\right]+\frac{3}{4}\left[w_{x}(i+1, j+1)+w_{x}(i-1, j-1)-w_{x}(i-1, j+1)-w_{x}(i+1, j-1)\right]+ \\
& w_{y}(i+1, j)+w_{y}(i-1, j) \\
& T_{x}=T(i+1, j)-T(i-1, j)
\end{aligned}
$$

The dimensionless parameter $\lambda$ is a ratio of the intra- and extracellular shear moduli

$$
\lambda=\frac{\mu}{v}
$$

Equation 26. 
$\sigma$ is a length constant characteristic of the bidomain model $^{3}$

$$
\sigma=\sqrt{\frac{v \mu}{K(v+\mu)}},
$$

and $\Delta$ is the space step between adjacent grid points. Boundary conditions were implemented by using a layer of fictitious nodes along the boundaries. These equations were solved iteratively for $M$ iterations using the software Octave. In the simulations $N_{x}=$ $N_{y}=103$ and $M \approx 10000$.

\section{RESULTS}

Fixed Boundary

The fixed boundary case simulates a sheet of tissue that has all corners and edges constrained such that they cannot undergo displacement, but the tissue is still subjected to a tension T. Figure 1 shows the intra- and extracellular displacements, $\mathbf{u}$ and $\mathbf{w}$.

(a)

(b)

Figure 1. (a) The intracellular displacement $\mathbf{u}$ and (b) extracellular displacement $\mathbf{w}$, for a fixed boundary.

The fiber tension acts in the horizontal direction (the direction of the myocardial fibers) and results in the ischemic border zone being stretched outwards away from the center. The intra- and extracellular displacements are similar.

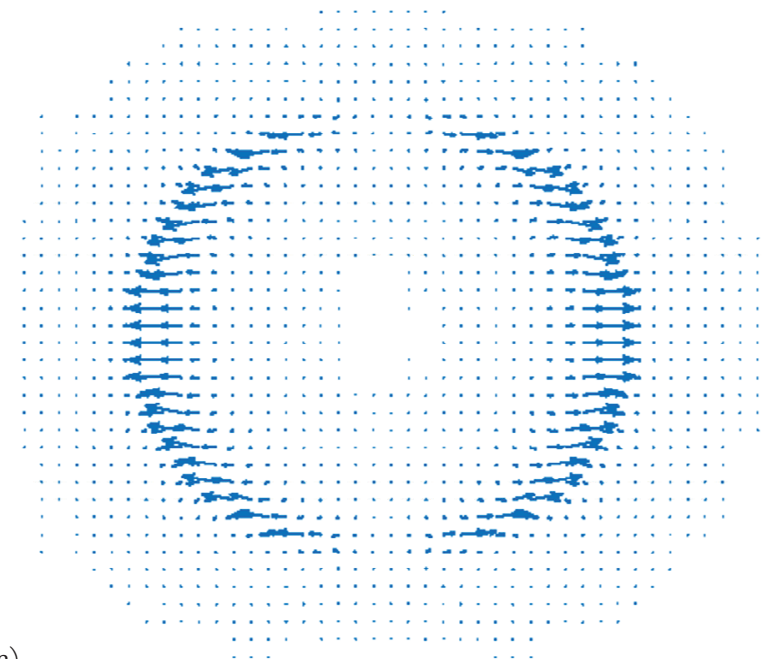

(a) 
(b)
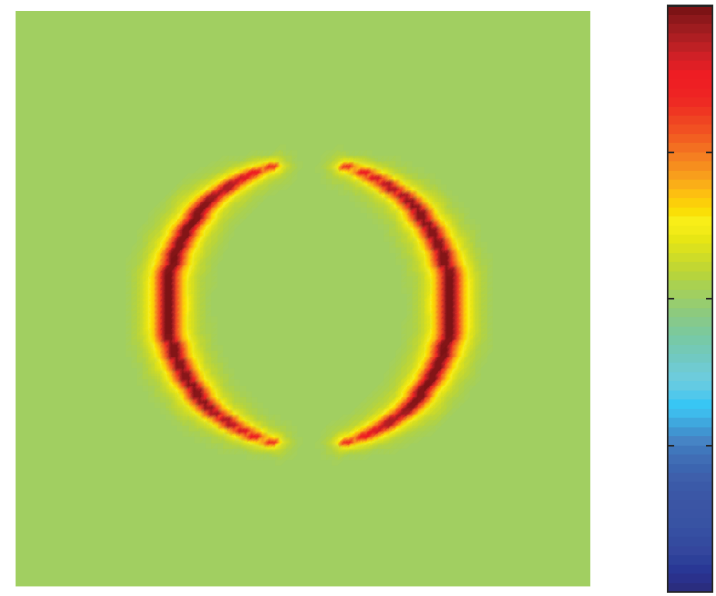

0.001

0

$-0.001$

Figure 2. (a) The difference $\mathbf{u}-\mathbf{w}$ and (b) the magnitude of the difference $|\mathbf{u}-\mathbf{w}|$, for a fixed boundary.

Figure 2 shows the difference between $\mathbf{u}$ and $\mathbf{w}$. Figure 2a is scaled differently than Figure 1; the largest arrow in Figure 1 has a magnitude that is approximately 4 times larger than the magnitude of the largest arrow in Figure 2. The difference between $\mathbf{u}$ and $\mathbf{w}$ is large only in the border zone, whereas $\mathbf{u}$ and $\mathbf{w}$ individually extend into the ischemic and healthy tissue. The difference $\mathbf{u}-\mathbf{w}$ is largest in the horizontal direction parallel to the myocardial fibers, and is zero perpendicular to them.

(a)

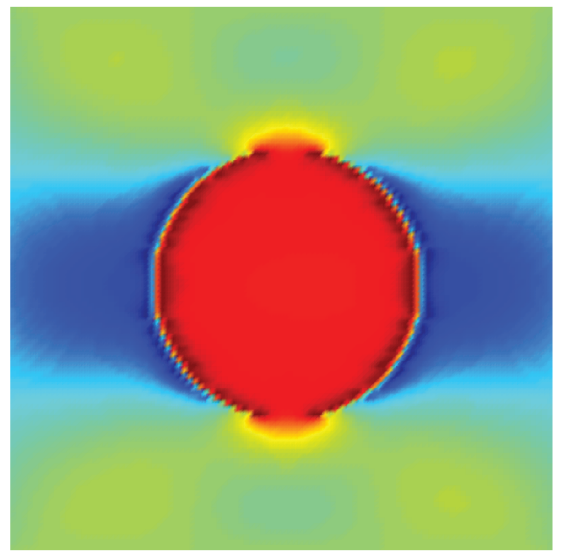

(c)

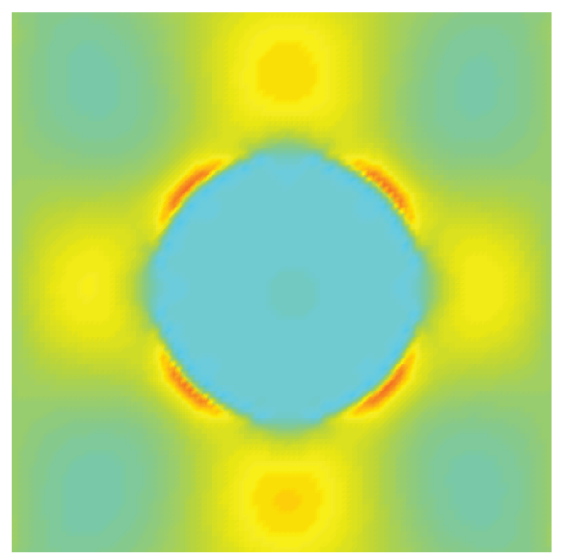

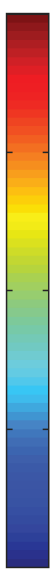

0.05

0

$-0.05$

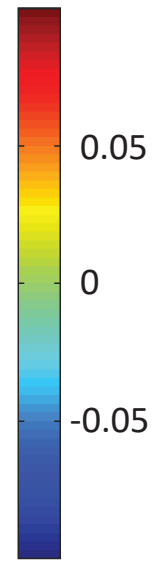

(b)
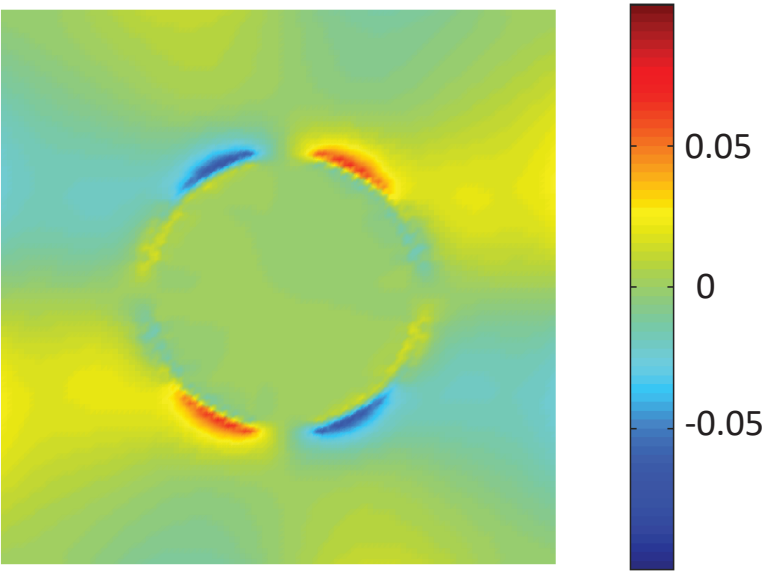

0.05

(d)
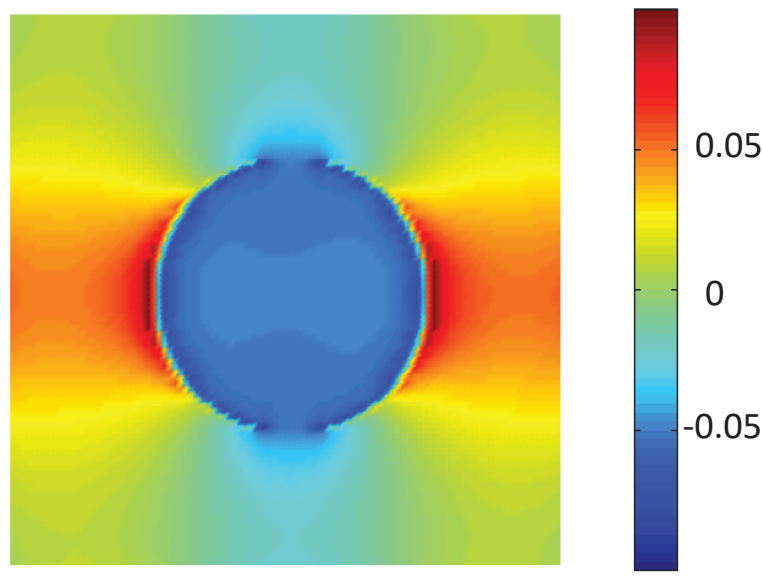

Figure 3. (a) The normal strain $\left(\varepsilon_{\mathrm{ixx}}\right)$, (b) shear strain $\left(\varepsilon_{\mathrm{ixy}}\right)$, (c) the $y$-strain $\left(\varepsilon_{\mathrm{iyy}}\right)$, and (d) the - $_{\text {-strain }}\left(\varepsilon_{\mathrm{izz}}\right)$ for a fixed boundary. 
The strain seems to be largest in the ischemic zone and outside the ischemic region along the fiber direction (Figure 3). The displacement difference distribution in Figure 2 does not match the distribution of the strains in Figure $\mathbf{3}$ which indicates that the mechanical bidomain model predicts that mechanotransduction occurs at different locations depending on if strain or membrane force is the causing factor.

Free Boundary

The free boundary case simulates a sheet of tissue that is able to undergo displacement anywhere along the tissue edge. Figure 4 shows the displacements $\mathbf{u}$ and $\mathbf{w}$. The difference in displacements, $\mathbf{u}-\mathbf{w}$, is virtually zero everywhere except at the vertical edges and in the ischemic border zone (Figure 5). The intracellular strains are shown in Figure 6.

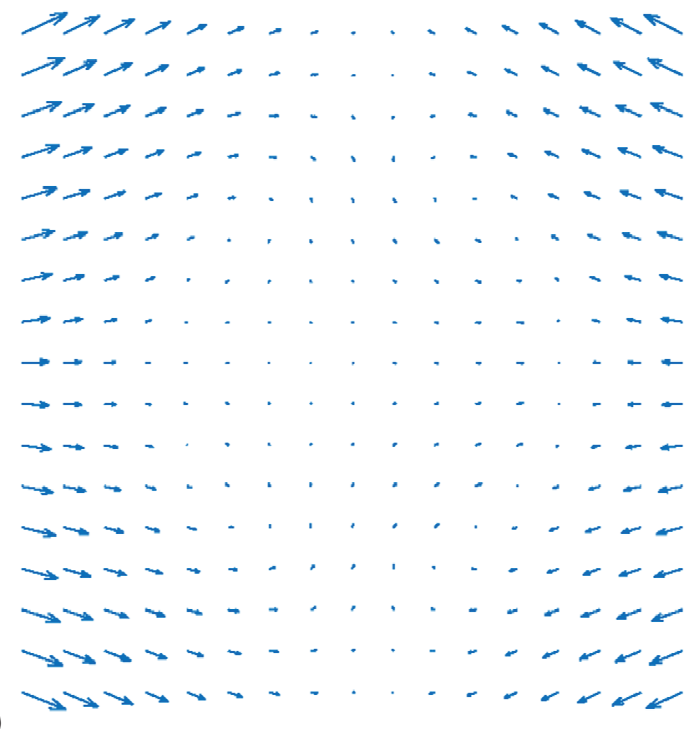

(a)

Figure 4. (a) The intracellular displacement $\mathbf{u}$ and (b) the extracellular displacement $\mathbf{w}$, for a free boundary.

(b)

The displacements $\mathbf{u}$ and $\mathbf{w}$ contract inwards to the ischemic region unlike the displacements in Figure 1.

(a)

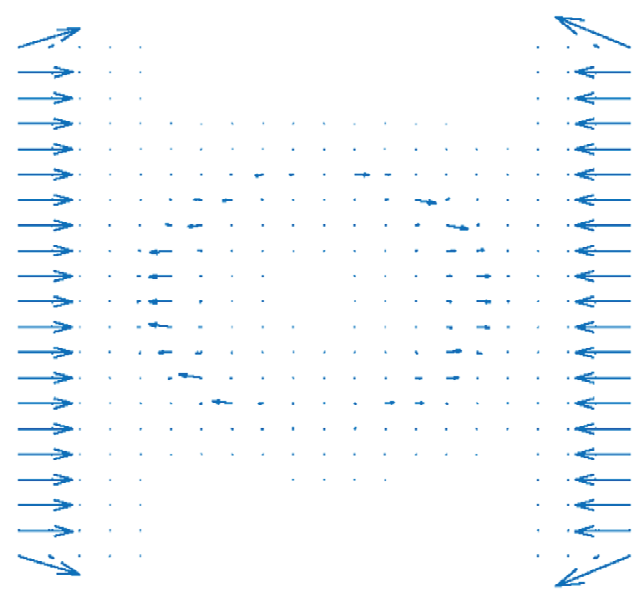

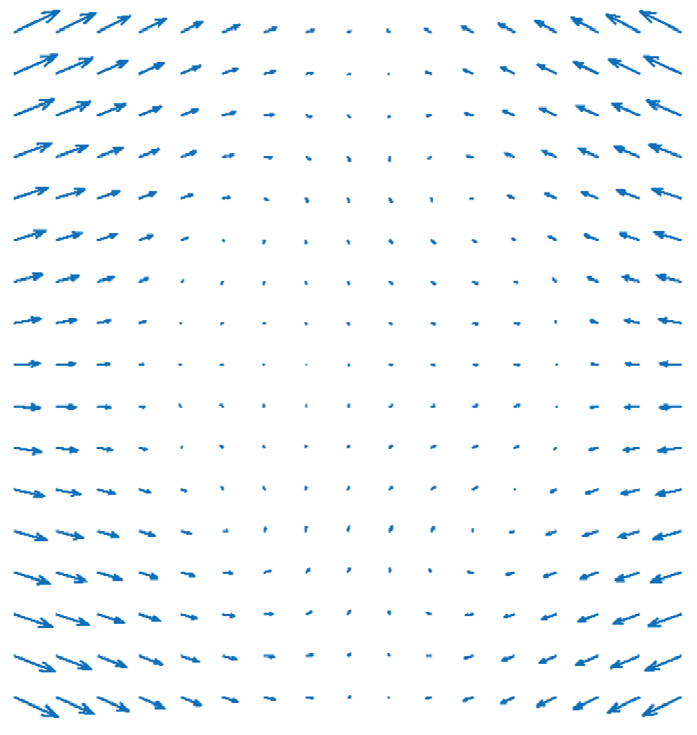

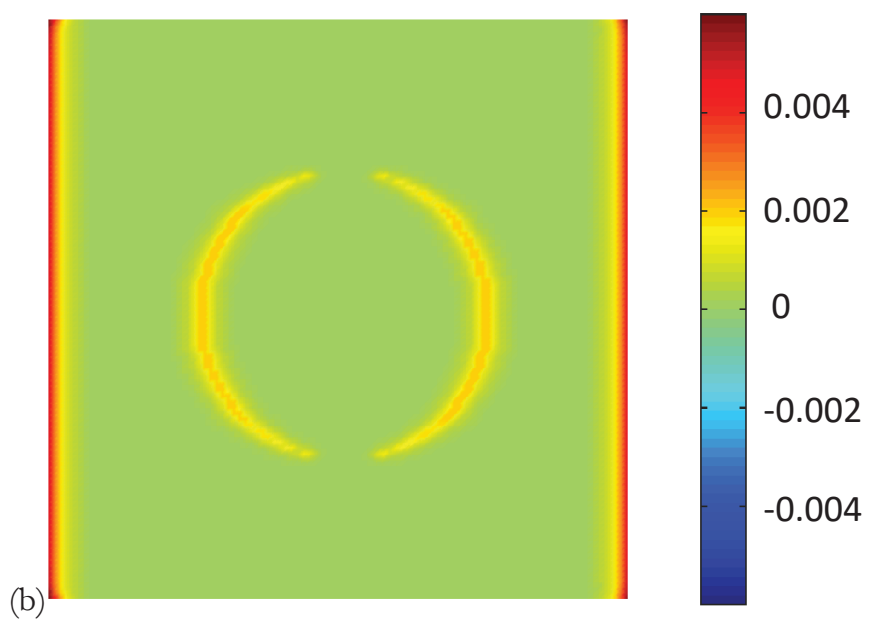

Figure 5. (a) The difference $\mathbf{u}-\mathbf{w}$. (b) The magnitude of the difference $|\mathbf{u}-\mathbf{w}|$, for a free boundary. 
(a)
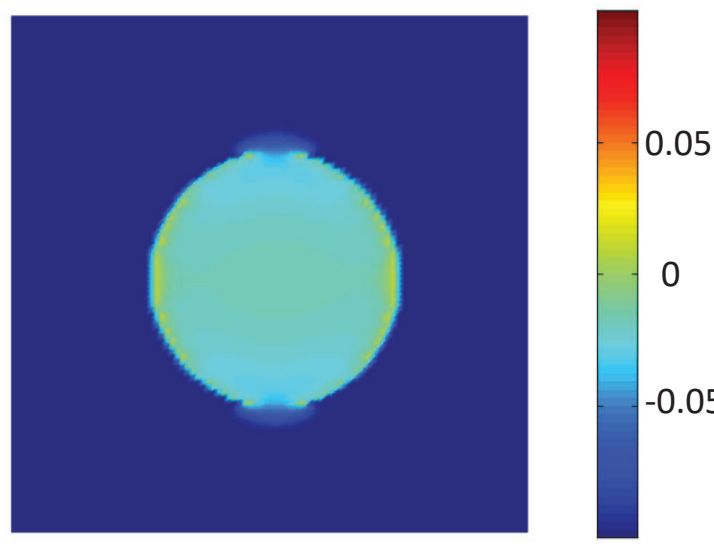

(b)

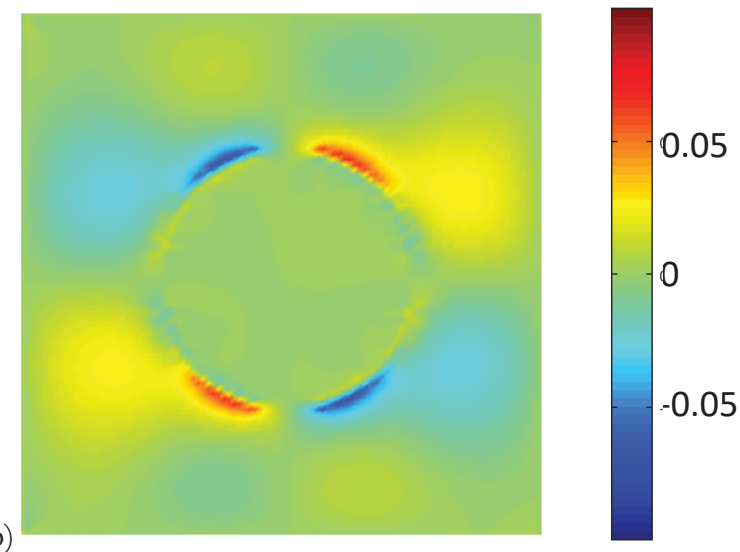

(d)

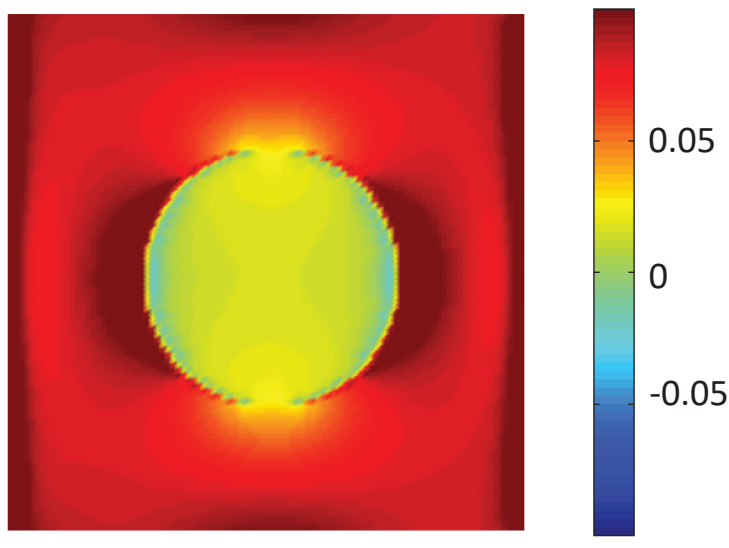

Figure 6. (a) The normal strain $\left(\varepsilon_{\mathrm{ixx}}\right)$, (b) the shear strain $\left(\varepsilon_{\mathrm{ixy}}\right)$, (c) the $y$-strain $\left(\varepsilon_{\mathrm{iyy}}\right)$, and (d) the $z_{-}$-strain $\left(\varepsilon_{\mathrm{izz}}\right)$ for a free boundary.

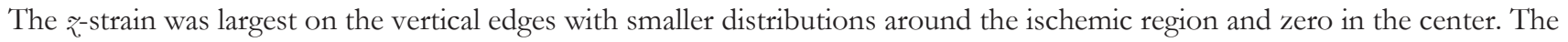
distribution of the $z_{\text {-strain }}$ is similar to the distribution for the membrane displacement differences along the ischemic region and borders as observed in Figure 5, but with a more complicated pattern outside the ischemic region.

\section{DISCUSSION}

A previous calculation based on the mechanical bidomain model for an ischemic region in cardiac tissue indicated that stress and strain were distributed widely throughout the normal and ischemic tissue, while the difference in intra- and extracellular displacements was restricted to the ischemic border zone. ${ }^{6}$ The localization of tissue growth and remodeling to the border zone was observed clinically. ${ }^{8}$ Gandhi and Roth's calculation was based on plane strain: no displacements in the $z$-direction. In this calculation, we compare their results with a similar calculation based on plane stress: no stress in the z-direction. In addition, Gandhi and Roth only considered the case of a fixed outer boundary. In our simulations, we consider both a fixed boundary and a free boundary.

Our results for a fixed boundary (Figures 1-3) can be directly compared to Gandhi and Roth's results. In both cases, the normal tissue was displaced toward the left and right sides of the tissue sheet outside of the ischemic region along the fiber direction. However, Gandhi and Roth also observed an inward movement of the displacement outside the ischemic region perpendicular to the fibers, caused by incompressibility. This inward movement was not as pronounced in our simulations (Figure 1) because with plane stress, incompressibility could be enforced by the tissue getting thinner (displacement in the z-direction) as well as the tissue moving inward. Figure 3d shows a negative strain in the z-direction within the ischemic region, indicating tissue thinning. In both our results (Figure 2) and those of Gandhi and Roth, the difference $\mathbf{u}-\mathbf{w}$ was concentrated in the border zone separating the ischemic and normal tissue regions. However, the spatial distribution was slightly different for our calculation of $\mathbf{u}-\mathbf{w}$ compared to that calculated by Gandhi and Roth. ${ }^{6}$ Gandhi and Roth found that $\mathbf{u}-\mathbf{w}$ was zero where the border zone intersected the $x$ axis, while $\mathbf{u}-\mathbf{w}$ was largest there in our results. Also, the direction of $\mathbf{u}-\mathbf{w}$ changed; Gandhi and Roth found it to be tangential to the border zone, whereas we found it to be parallel to the $x$-axis. Both calculations found large positive strains $\varepsilon_{\mathrm{ixx}}$ in the ischemic region, but Gandhi and Roth found large negative strains in the normal tissue both parallel and perpendicular to the 
fibers, whilst our results showed only negative strains in the normal tissue parallel to the fibers (Figure 3). Therefore, we conclude significant differences exist in the displacement and strain distributions between plane stress and plane strain, although both cases restrict the difference $\mathbf{u}-\mathbf{w}$ to the ischemic border zone.

When we allowed the tissue to have a free boundary (Figures 4-6), we found additional new behavior compared to the fixed boundary. For instance, the displacements $\mathbf{u}$ and $\mathbf{w}$, and the intracellular strain $\varepsilon_{\mathrm{ixx}}$, were nearly zero throughout the ischemic tissue. The surrounding normal tissue contracted (Figure 4), but it did not cause the ischemic region to move because there was no fixed boundary to pull on. Unlike the fixed boundary, the tissue expanded in the ₹-direction indicated by a positive z-strain to obey incompressibility as the tissue contracted. In addition, near the left and right edges of the normal tissue there was a thin boundary layer of $\mathbf{u}-\mathbf{w}$. Such boundary layers have been observed in previous calculations, such as that by Roth. ${ }^{9}$ This boundary layer fell off with distance from the boundary exponentially with length constant $\sigma$. Therefore, for a free boundary, $\mathbf{u}-\mathbf{w}$ was restricted to two regions: the ischemic border zone, and the tissue outer edge. The free boundary strains correlated insignificantly to Gandhi and Roth's fixed boundary strains. They found complex geometries outside the ischemic region where our strain distributions were fairly simple and saturated. Our free $\mathbf{u}-\mathbf{w}$ compared to their results similarly as our fixed $\mathbf{u}-\mathbf{w}$, but with the addition of displacement differences on the outer edge. These comparisons further support the difference in strain and membrane force distribution depending on whether plane strain or plane stress is used as well as what boundary conditions are in effect.

The numerical method used in this study differs significantly from that used by Gandhi and Roth. ${ }^{6}$ In the case of plane strain, the condition of incompressibity implies that $\frac{\partial u_{x}}{\partial x}+\frac{d u_{y}}{d y}=0$, and this condition can be enforced by using stream functions to specify $\mathbf{u}$ and $\mathbf{w}$. For plane stress, the stream functions are no longer useful, and the differential equations of the bidomain model had to be written in terms of displacements instead. Our results are independent of the thickness of the tissue sheet, as long as it is thin enough that the plane stress assumption is applicable. We are primarily interested in the spatial distribution of the strains and membrane forces, rather than their magnitude, so we set $T=1$. The model is linear, so results for other values of $T$ could be found by linear scaling.

These results have implications for how cardiac tissue recovers from a heart attack. Rodriguez et al. found that mechanical alternations such as remodeling following a heart attack occurs primarily in the ischemic border zone. ${ }^{8}$ These results are consistent with our prediction that membrane forces are largest in the border zone.

The plane stress case is useful when analyzing data from experiments on cell monolayers because it simulates a two-dimensional sheet like our model. The free and fixed boundary conditions can be simulated on such monolayers such that plane stress can predict where mechanotransduction will occur. ${ }^{4,5}$ Rosowski et al. performed such an experiment on a cell monolayer with a free upper surface, but not a fixed one, and found that cells primarily differentiated at the edge. ${ }^{10}$ Our model predicted similar results to this behavior. Future experiments simulating a fixed boundary with an ischemic region would offer evidence testing the predictions found using this model.

The model used contained various limitations:

1. The ratio between the intra- and extracellular shear moduli was assumed to be one.

2. The actual value for the length constant is not known.

3. While the z-direction is considered in this analysis, it still does not represent a general three-dimensional solution which would be the ideal model for actual cardiac tissue.

4. Linear stress-strain and strain-displacement relationships are assumed when nonlinearities may exist.

5. Straight fiber geometries are used when realistic cardiac tissue contains curved fiber geometries. ${ }^{7}$

6. The ischemic border zone is abrupt and thin. Actual ischemic borders may be irregular in heart tissue.

In this research, mechanotransduction, under fixed boundaries, was found to reside in the border zone of the ischemic region parallel to fiber orientation. This result is similar to where plane strain models found mechanotransduction to occur under fixed boundaries, but with slight differences in distribution. ${ }^{6}$ However, the individual displacement distributions of the intracellular and extracellular spaces between the plane stress and plane strain models differed. These results show that the plane stress and plane strain models localize mechanotransduction to the ischemic border zone, but the distributions of the individual cellular displacements and mechanotransduction differ depending on which model is used. Additionally, the localization of mechanotransduction using plane stress differed depending on if the boundaries where fixed or free showing that boundary conditions affect where cells will grow or remodel on a sheet of tissue. If strain is assumed to be the cause of mechanotransduction then this research found that the strain distributions throughout the sheet of tissue differed depending on if plane strain or plane stress was used. Plane strain, under fixed boundaries, found a more complex distribution of strain throughout the normal tissue than the plane stress model. Furthermore, the distribution of strain under plane stress differed depending on if fixed or free boundary conditions were implemented. 


\section{CONCLUSIONS}

In conclusion, the mechanical bidomain model found mechanotransduction to occur in the ischemic border zone assuming membrane force as the cause of mechanotransduction while the same model found mechanotransduction to occur throughout the ischemic region and healthy tissue assuming strain as the cause of mechanotransduction. The mechanical bidomain model predicts where mechanotransduction occurs differently depending on whether membrane forces or strains are assumed to induce mechanotransduction. This research may provide valuable insight into understanding how cardiac tissue recovers after a heart attack. Mechanotransduction in cardiac tissue may lead to cellular remodeling. ${ }^{7}$ The mechanical bidomain model can be used to describe the remodeling process and predict where the remodeling will occur. These applications of the mechanical bidomain model may aid in describing and understanding in vitro and in vivo experiments dealing with ischemic myocardium. To determine whether cellular strain or membrane force causes mechanotransduction and whether plane stress or plane strain provides a better model for simulating mechanotransduction, more quantitative experiments need to be conducted. The free boundary conditions under plane strain have yet to be analyzed using the mechanical bidomain model. Quantitative evidence for a sheet of tissue with active tension with a central circular ischemic region is lacking. If the conditions used in this research where replicated in a lab and analyzed the results would help determine whether plane stress or plane strain is the better model for predicting mechanotransduction. Additionally, this evidence would also help in determining whether strain or membrane force is the causing factor of mechanotransduction. This experiment could be performed in a lab if a sheet of healthy cardiac tissue was restricted between two coverslips, to simulate plane strain, and was electronically stimulated to replicate active tension. The ischemic region could be induced through drug applications. The plane stress conditions could be replicated if the top coverslip was removed such that the tissue could displace in the z-direction. Fixed boundary conditions could be implemented by pinning the edges of the tissue down so that no edge displacements can occur. Free boundary conditions can be implemented by not pinning the edges of the tissue so that the edges can undergo displacement. Finally, the mechanical bidomain model is only two-dimensional. A three-dimensional model may provide a better representation of mechanotransduction in physical myocardium, but such a model has not yet been derived.

\section{ACKNOWLEDGEMENTS}

The authors thank the Oakland University Summer Undergraduate Research Program in the Biological Sciences and Chemistry Departments.

\section{REFERENCES}

1. Dabiri, B. E., Lee, H., \& Parker, K. K. (2012). A potential role for integrin signaling in mechanoelectrical feedback. Prog Biophys Mol Bio,110(2-3), 196-203. doi:10.1016/j.pbiomolbio.2012.07.002

2. Kresh, J. Y., \& Chopra, A. (2011). Intercellular and extracellular mechanotransduction in cardiac myocytes. Pflug Arch Eur J Phy,462(1), 75-87. doi:10.1007/s00424-011-0954-1

3. Roth, B. J. (2016). A Mathematical Model of Mechanotransduction. arXiv:1611:08287.

4. Auddya, D., \& Roth, B. J. (2017). A mathematical description of a growing cell colony based on the mechanical bidomain model. J Phys D Appl Phys,1-7. doi:10.1088/1361-6463/aa59b5

5. Scribner, C., \& Roth, B. J. (2017). Plane strain versus plane stress in the mechanical bidomain model. Meeting of Minds Journal of Undergraduate Research. (In press)

6. Gandhi, S., \& Roth, B. J. (2015). A numerical solution of the mechanical bidomain model. Comput Method Biomec Biomed Eng,19(10), 1099-1106. doi:10.1080/10255842.2015.1105964

7. Sharma, K. (2018). The mechanical bidomain model of cardiac tissue. PhD dissertation, Oakland University, Rochester, MI.

8. Rodriguez, F., Langer, F., Harrington, K. B., Cheng, A., Daughters, G. T., Criscione, J. C., . . Miller, D. C. (2005). Alterations in transmural strains adjacent to ischemic myocardium during acute midcircumflex occlusion. J Thorac Cardiov Sur,129(4), 791803. doi:10.1016/j.jtcus.2004.11.011

9. Roth, B. J. (2013). Boundary layers and the distribution of membrane forces predicted by the mechanical bidomain model. Mech Res Commun, 50, 12-16. doi:10.1016/j.mechrescom.2013.02.004

10. Rosowski, K. A., Mertz, A. F., Norcross, S., Dufresne, E. R., \& Horsley, V. (2015). Edges of human embryonic stem cell colonies display distinct mechanical properties and differentiation potential. Sci Rep,5(1), 14218. doi:10.1038/srep14218 


\section{ABOUT STUDENT AUTHOR}

Austin Fee is currently an undergraduate junior student pursuing a B.S. in Bioengineering at Oakland University. He plans on attending graduate school to obtain a M.S. in Mechatronics. He intends to pursue a research career in robotic prostheses.

\section{PRESS SUMMARY}

Mechanotransduction is a tissue's biological reaction to a mechanical force. An example of mechanotransduction, relevant to this study, is how cardiac tissue regrows and changes (remodels) following a heart attack. In previous studies, mechanotransduction was thought to be caused by the stretching or shearing of tissue (strain). For this study, mechanotransduction is assumed to be caused by differences in displacements between the intracellular and extracellular spaces producing a force on integrin proteins in the cell membrane. To describe and predict mechanotransduction in tissue sheets, a mathematical model was developed. Earlier studies using a similar model to examine a two-dimensional sheet of tissue assumed the tissue could not move in the direction perpendicular to the sheet (plane strain). This paper examines a different case when the tissue experiences no force perpendicular to the sheet (plane stress). The plane stress case may be a better description of experiments performed on sheets of tissue one cell thick (monolayers). The model predicts that mechanotransduction occurs mainly in the border zone between healthy and unhealthy tissue, and the exact distribution of mechanotransduction differs between the plane stress and plane strain cases. 